\title{
Efectos del Albendazol en el Hígado de Feto de Rata. Estudios Morfológico y Morfométrico
}

\author{
Effects of Albendazole on Rat Fetuses Liver. \\ Morphologic and Morphometric Studies \\ "Dora Lúcia Carrara Moreti; ", *** Ruberval Armando Lopes; "Dionisio Vinha; "**Miguel Angel Sala; *"Marisa Semprini \& \\ ${ }^{* *}$ Christiane Friedrichi
}

MORETI, D. L. C.; LOPES, R. A.; VINHA, D.; SALA, M. A.; SEMPRINI, M. \& FRIEDRICHI, C. Efectos del albensazol en el hígado de feto de rata. Estudios morfológico y morfométrico. Int. J. Morphol., 23(2):111-120, 2005.

RESUMEN: El objetivo del presente trabajo fue caracterizar morfológica, morfométrica y estereológicamente las alteraciones causadas por la administración de albendazol en ratas preñadas en el hígado de los fetos. Fueron usadas ratas Wistar, que recibieron entre los días 9 y 11 de la preñez, una dosis diaria de $5 \mathrm{mg} / \mathrm{kg}$ de peso de albendazol. Las ratas control recibieron volumen semejante de suero fisiológico en los mismos días. Al $20^{\circ}$ día, las ratas fueron sacrificadas por inhalación de éter, siendo retirados los fetos e inmediatamente fijados en alfac por $24 \mathrm{~h}$. Los fetos, úteros y placentas, luego de fijados, fueron secados en papel de filtro y pesados en balanza de precisión, siendo medidos los cordones umbilicales con nonio. Los cuerpos de los fetos fueron seccionados longitudinalmente e incluidos en parafina. Los cortes seriados de $6 \mu \mathrm{m}$ de espesor, fueron teñidos con hematoxilina y eosina. Además del examen histopatológico, fueron utilizadas técnicas histométricas. Los resultados obtenidos, en las condiciones experimentales, sugieren que la exposición prenatal al albendazol actúa directamente en la embriogénesis, causando malformaciones como agenesia del rabo y fetos hidrópicos. En los fetos sin malformaciones, la droga causó retardo del desarrollo fetal, caracterizado por reducción de peso corporal, peso de la placenta y longitud del cordón umbilical, así como alteraciones estructurales hepáticas, reflejadas en hepatocitos menores con núcleos menores y citoplasma más escaso y aumento de la densidad numérica. Los megacariocitos eran mayores, con núcleos lobulados grandes, con mayor volumen relativo. Los sinusoides ocuparon un volumen relativo semejante en los fetos de los dos grupos estudiados. Los fetos tratados, además de las malformaciones, mostraron aspectos que sugieren inmadurez al ser comparados con los controles.

PALABRAS CLAVE: Hígado; Feto; Rata; Albendazol; Morfometría.

\section{INTRODUCCIÓN}

Los benzimidazoles son antihelmínticos de reconocida eficacia contra varios parásitos del tracto gastrointestinal. Sus compuestos presentan efectos teratogénicos en animales domésticos y de laboratorio, siendo la rata particularmente susceptible a los efectos teratógenos y embriocidas (Lapras et al., 1973; Duncan \& Lemon, 1974; Delatour et al., 1982).

El albendazol (ABZ) es un benzimidazol rápidamente metabolizable y su principal metabolito, el sulfóxido de albendazol (SOABZ), también denominado ricobendazol, es un potente antihelmíntico utilizado extensamente tanto en animales como en humanos (Zhangliu et al., 1992; Lanusse \& Pritchard, 1993).
El ABZ y el SOABZ han sido usados como modelos para elucidar la inducción de dismorfogénesis provocadas por los benzimidazoles, habiendo sido demostrado en ovinos (Marriner, 1986; Fabre et al., 1989; McKellar \& Scott, 1990), vacunos (Delatour et al., 1981; Piscopo \& Smoak, 1997) y ratas (Mantovani et al., 1995; Cristófol et al.,1997; Piscopo \& Smoak; Navarro et al., 1999; Teruel et al., 2003). La actividad teratógena del ABZ in vivo es atribuida al SOABZ, debido a su mayor concentración sistémica cuando es comparada a la del ABZ (Delatour et al., 1984).

El objetivo de este trabajo es evaluar y cuantificar las alteraciones hepáticas presentes en fetos de ratas sometidas a la acción del ABZ.

\footnotetext{
* Universidade de Franca, São Paulo, Brasil.

** Departamento de Morfología, Estomatología y Fisiología de la Facultad de Odontología de Ribeirão Preto, Universidade de São Paulo, Brasil. Disertación de Magíster del primer autor, presentada a la Universidade dee Franca (Área de Concentración en Promoción de Salud).
} 


\section{MATERIAL Y MÉTODO}

Fueron usadas 14 ratas, variedad Wistar, con peso medio de $170 \mathrm{~g}$. Las ratas fueron alojadas en jaulas individuales y alimentadas con ración comercial y agua ad libitum.

Las ratas fueron colocadas para cruzarse con los machos durante la noche, considerándose como el primer día de la preñez, si se verificada la presencia de espermatozoides en el frotis vaginal.

En los días 9, 10 y 11 de la preñez, 7 ratas recibieron 5 $\mathrm{mg} / \mathrm{kg} /$ día de Albendazol (Zolben®), Sanofi Pharma do Brasil), vía oral. Otras 7 ratas recibieron suero fisiológico y sirvieron de control. En el $20^{\circ}$ día de preñez, las ratas fueron sacrificadas por inhalación de éter y, a través de una incisión amplia del abdomen y útero, fueron retirados los fetos y anexos. Después de 24 h de fijación en alfac, los fetos y las placentas fueron pesados en balanza de precisión Mettler y el cordón umbilical medido con un nonio. El líquido amniótico fue estimado por la diferencia entre los pesos del útero y de los anexos.

Los cuerpos de 5 fetos del grupo tratado y 5 del grupo control, escogidos al azar de un total de 76 tratados y 84 controles, fueron seccionados longitudinalmente y deshidratados, diafanizados e incluidos en parafina. Posteriormente, fueron realizados cortes seriados de $6 \mu \mathrm{m}$ de espesor, de los cuales se separaron 10 cortes por bloque, cada uno de los cuales correspondió, así, a un intervalo de 50 cortes. Los cortes histológicos fueron teñidos con hematoxilina y eosina.

Para el análisis morfométrico, los cortes histológicos fueron examinados al microscopio óptico, con cámara clara, con objetivo de inmersión. Para obtención de los diámetros mayor y menor de los núcleos de los hepatocitos y megacariocitos, fueron realizados dibujos sobre papel, con aumento de 1000 x. Las imágenes nucleares obtenidas fueron contornadas con lápiz $\mathrm{N}^{\circ} 2$, considerándose solamente las figuras elípticas. A continuación, con la ayuda de papel milimetrado, fueron medidos los ejes mayor y menor de esas imágenes.

Fueron estimados los siguientes parámetros nucleares: diámetro medio, relación diámetro mayor/diámetro menor, perímetro, área, volumen, relación volumen/área, excentricidad, coeficiente de forma e índice de contorno (Sala et al. , 1994).

El análisis estereológico fue realizado utilizando una retícula curvilínea (Merz, 1968), impresa sobre papel, donde fueron dibujadas las estructuras histológicas del hígado. Los siguientes parámetros fueron estimados: volúmenes citoplasmático y celular, relación núcleo-citoplasma y densi- dad numérica (Sala et al., 1992). Los volúmenes relativos fueron determinados mediante la técnica de Chalkley (1943).

La comparación estadística fue realizada mediante la prueba no paramétrica de Mann-Whitney.

\section{RESULTADOS}

El peso corporal materno de las ratas controles fue igual a 428,2 g y el de las ratas tratadas con ABZ fue de 299,0 $\mathrm{g}$. El peso medio del útero de las ratas tratadas fue de $3,3 \mathrm{~g}$, mientras que en los animales del grupo control de 4,8 g. En las ratas tratadas, el líquido amniótico fue más escaso que en las ratas del grupo control $(26,12 \mathrm{mg}$ y $38,12 \mathrm{mg}$, respectivamente).

Fue observada una reducción altamente significativa en los pesos de los fetos del grupo tratado comparados con los del grupo control (Tabla I). El peso medio de la placenta también fue significativamente menor en el animal tratado que en el control (Tabla I). El cordón umbilical fue más corto en los fetos tratados que en los controles.

Tabla I. Valores morfométricos medios de los fetos de las ratas de los grupos control y tratado con albendazol. Prueba de MannWhitney. (P[U]: probabilidad)

\begin{tabular}{lrrrc}
\hline Parámetros & Control & Tratado & U calc & $\mathrm{P}[\mathrm{U}]$ \\
Peso fetal $(\mathrm{g})$ & 4,29 & 3,18 & $0^{*}$ & 0,004 \\
Peso de la placenta $(\mathrm{mg})$ & 577,10 & 326,00 & $0^{*}$ & 0,004 \\
Longitud del cordón $(\mathrm{mm})$ & 2,80 & 1,60 & $0^{*}$ & 0,004 \\
\hline "p $<0,01$
\end{tabular}

En el estudio piloto se observó gran número de reabsorciones fetales, luego de la administración de $10 \mathrm{mg} /$ kg/día de ABZ, en los días 9, 10 y 11 de la preñez. Cuando la dosis administrada fue de $5 \mathrm{mg} / \mathrm{kg} /$ día, fue posible observar agenesia de la cola (40\%) y fetos hidrópicos (10\%).

Histológicamente, el hígado del feto de ratas controles muestra hepatocitos bien evidentes, con núcleos de cromatina laxa y citoplasma acidófilo, constituyendo placas pequeñas de células. Entre estas placas se observan los sinusoides. Son visibles células sanguíneas y megacariocitos (Figs. 1 y 2).

El tejido hepático de los fetos de ratas tratadas con ABZ se muestra desorganizado, con hepatocitos presentando degeneración hidrópica, con citoplasma granular y núcleos de menor tamaño. Los sinusoides se presentan dilatados y los linfocitos son más numerosos. Los megacariocitos se muestran más voluminosos. Algunas células están necrosadas y los núcleos en cariolisis (Figs. 3 y 4). 

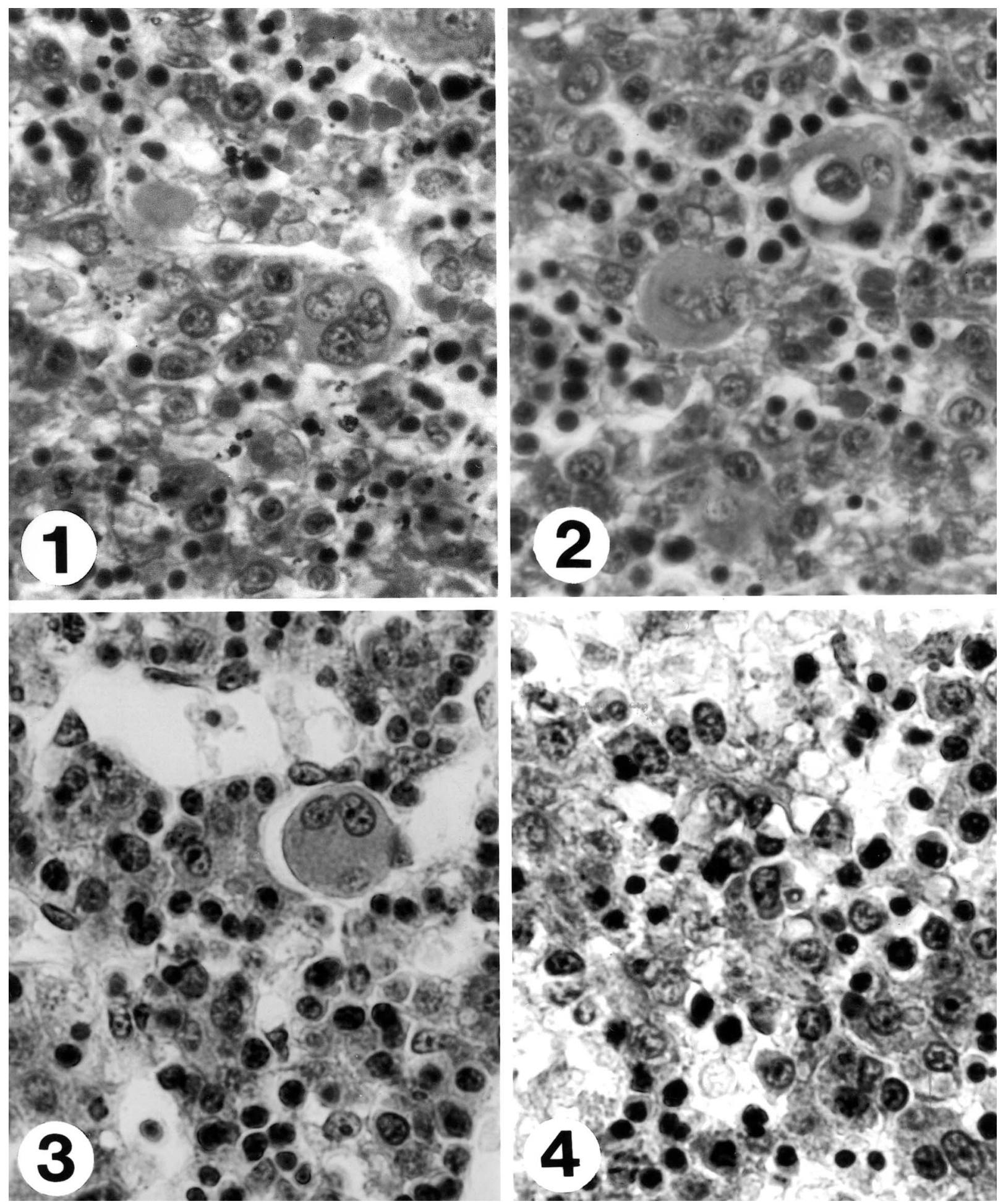

Fig. 1. Aspecto histológico del hígado de feto de rata control. Notar los hepatocitos más organizados, sinusoides, megacariocitos y otras células sanguíneas (eritrocitos y linfocitos). HE. 900 x.

Fig. 2. Aspecto histológico del hígado de feto de rata control. Notar los hepatocitos, sinusoides y células sanguíneas. HE. 900 x.

Fig. 3. Aspecto histológico del hígado de feto de rata tratada con albendazol. Notar los hepatocitos menores, megacariocitos voluminosos y demás células. HE. $900 \mathrm{x}$.

Fig. 4. Aspecto histológico del hígado de feto de rata tratada con albendazol. Notar desorganización celular y sinusoides dilatados. HE. 900 x.

Analizando la Tabla II se puede observar que los valores cariométricos medios para los diámetros mayor, menor y medio, perímetro, área, volumen y relación volumen/área son menores en los núcleos de los hepatocitos y mayores en los lóbulos nucleares de los megacariocitos de los fetos pertenecientes al grupo tratado. Los valores para los parámetros que evalúan la forma nuclear eran semejantes en los hepatocitos de los fetos de los dos grupos estudiados. En los megacariocitos 
los valores para excentricidad e índice de contorno son mayores en los fetos de las ratas tratadas con ABZ.

Tabla II. Valores cariométricos medios de los hepatocitos y megacariocitos (lóbulos) del hígado de fetos de ratas de los grupos control y tratado con albendazol. Prueba de Mann-Whitney.

\begin{tabular}{lcccc}
\hline \multicolumn{1}{c}{ Parámetros } & \multicolumn{2}{c}{ Control } & Tratado & \multicolumn{2}{c}{ Control } & Tratado \\
Diámetro mayor $(\mu \mathrm{m})$ & 7,17 & $6,17^{* *}$ & 7,12 & $8,07^{* *}$ \\
Diámetro menor $(\mu \mathrm{m})$ & 6,40 & $5,34^{* *}$ & 5,15 & $6,26^{* *}$ \\
Diámetro medio $(\mu \mathrm{m})$ & 6,76 & $5,74^{* *}$ & 6,02 & $7,08^{* *}$ \\
Relación D/d & 1,12 & $1,16^{* *}$ & 1,40 & $1,31^{* *}$ \\
Perímetro $(\mu \mathrm{m})$ & 21,33 & $18,16^{* *}$ & 19,46 & $22,65^{* *}$ \\
Área $\left(\mu \mathrm{m}^{2}\right)$ & 36,29 & $26,24^{* *}$ & 29,43 & $40,90^{* *}$ \\
Volumen $\left(\mu \mathrm{m}^{3}\right)$ & 167,11 & $103,22^{* *}$ & 125,39 & $208,06^{* *}$ \\
Relación V/A & 4,70 & $3,82^{* *}$ & 4,02 & $4,72^{* *}$ \\
Excentricidad & 0,35 & 0,39 & 0,60 & $0,54^{*}$ \\
Coeficiente de forma & 0,99 & 0,98 & 0,95 & 0,96 \\
Indice de contorno & 3,56 & 3,57 & 3,64 & $3,60^{*}$ \\
\hline
\end{tabular}

* $\mathrm{p}<0,05{ }^{* * *} \mathrm{p}<0,01$

El megacariocito presenta los diámetros mayor, menor y medio, además del perímetro, área y volumen, menores en los fetos del grupo tratado con ABZ, en comparación con los fetos del grupo control (Tabla III).

Tabla III. Valores morfométricos medios de los megacariocitos del hígado de fetos de ratas de los grupos control y tratado con albendazol. Prueba de Mann-Whitney.

\begin{tabular}{lcc}
\hline \multicolumn{1}{c}{ Parámetros } & Control & \multicolumn{1}{c}{ Tratado } \\
Diámetro mayor $(\mu \mathrm{m})$ & 22,57 & $23,71^{* *}$ \\
Diámetro menor $(\mu \mathrm{m})$ & 18,90 & $20,38^{*}$ \\
Diámetro medio $(\mu \mathrm{m})$ & 20,58 & $21,96^{* *}$ \\
Perímetro $(\mu \mathrm{m})$ & 65,40 & $69,43^{*}$ \\
Área $\left(\mu \mathrm{m}^{2}\right)$ & 342,24 & $386,05^{*}$ \\
Volumen $\left(\mu \mathrm{m}^{3}\right)$ & 4951,31 & $5865,02^{*}$ \\
\hline
\end{tabular}

$* \mathrm{p}<0,05 * * \mathrm{p}<0,01$

Mediante técnicas estereológicas se pudo observar que el volumen relativo del citoplasma de los hepatocitos es significativamente menor, mientras que el correspondiente a los núcleos es mayor en los fetos del grupo tratado comparados con el grupo control. El volumen relativo de los megacariocitos también está aumentado en estos fetos. Los valores correspondientes a sinusoides y linfocitos no muestran diferencias significativas (Tabla IV).

Tabla IV. Valores relativos medios (\%) de las estructuras hepáticas de fetos de ratas de los grupos control y tratado con albendazol. Prueba de Mann-Whitney.

\begin{tabular}{lrc}
\hline Estructuras & Control & Tratado \\
Hepatocito & & \\
Citoplasma & 30,72 & $20,44^{* *}$ \\
Núcleo & 9,47 & $17,76^{* *}$ \\
Sinusóides & 58,63 & 63,81 \\
Megacariocitos & 0,30 & $0,51^{*}$ \\
Linfocitos & 0,88 & 1,48 \\
\hline${ }^{*} \mathrm{p}<0,05^{* *} \mathrm{p}<0,01$ & &
\end{tabular}

Cuando se analiza la Tabla V, se puede verificar la existencia de valores significativamente menores para los volúmenes citoplasmático y celular, junto con aumento de la relación N/C y de la densidad numérica de los hepatocitos en los fetos de ratas tratadas con ABZ.

Tabla V. Valores estereológicos medios de los hepatocitos de fetos de ratas de los grupos control y tratado con albendazol. Prueba de Mann-Whitney.

\begin{tabular}{lrc}
\hline \multicolumn{1}{c}{ Parámetros } & Control & Tratado \\
Volumen citoplasmático $\left(\mu \mathrm{m}^{3}\right)$ & 1439,04 & $464,41^{*}$ \\
Volumen celular $\left(\mu \mathrm{m}^{3}\right)$ & 1606,15 & $567,63^{*}$ \\
Relación N/C & 0,1123 & 0,2134 \\
Densidad numérica $\left(\mathrm{n} / \mathrm{mm}^{3}\right)$ & 623346 & $1792963^{*}$ \\
\hline
\end{tabular}
${ }^{*} \mathrm{p}<0,01$

\section{DISCUSIÓN}

Todas las ratas permanecieron saludables, sin producirse muertes durante el experimento. Lo mismo fue observado en ratas tratadas con tiabendazol (Khera et al., 1979) o fluorbendazol (Yoshimura, 1987a) y en ratas tratadas con metiltiofanato (Makita et al., 1973). Mientras tanto, Ogata et al. (1984) observaron muerte de ratas preñadas después de administración oral de 1300 a 2400 mg/kg/día de tiabendazol.

Los efectos adversos de los benzimidazoles sobre el reducción de peso corporal durante la preñez, fueron descritos en ratas (Kavlock et al., 1982; Yoshimura, 1987b), ratones (Ogata et al., 1984) y conejas (Lankas \& Wise, 1993). En el presente estudio se observó disminución de peso corporal materno en las ratas tratadas con ABZ, probablemente debido a la reducción del peso de los fetos, placentas, cordones umbilicales y útero, y a la disminución del líquido amniótico.

El peso corporal medio de los fetos del grupo tratado fue significativamente menor, como se observó en ratas que recibieron $200 \mathrm{mg} / \mathrm{kg} /$ día de benomyl, en los días 7-17 de la gestación y en las ratas que recibieron 125 a 500 mg/kg/día en la dieta (Kavlock et al.). Peso fetal menor también fue observado con tiabendazol, en ratones (Ogata et al.,1984) y con flubendazol (Yoshimura, 1987a), triclabendazol (Yoshimura, 1987b) o mebendazol (Baskerville et al.,1988; Bombonato et al.,1994) en ratas.

La placenta de las ratas tratadas era significativamente menor que la placenta de las ratas controles. Se sabe que las drogas y otros fármacos atraviesan rápidamente la placenta y alcanzan al feto en desarrollo. Mientras tanto, los mecanismos responsables por el proceso de transferencia son poco conocidos (Goldstein et al., 1968). Además, la placenta su- 
fre alteraciones morfológicas durante la gestación, acompañadas por modificaciones de la permeabilidad (Huggett \& Hammond, 1952).

El ABZ posee un efecto vasoconstrictor sobre el flujo sanguíneo placentario (Kirkinem et al., 1983). A pesar que el flujo sanguíneo umbilical del feto no se altera por la acción del ABZ, se observó una disminución del flujo intervellositario de la placenta, en respuesta a la ingestión de la droga por la rata preñada. Se suma a esto, una elevación de los niveles séricos maternos de epinefrina $30 \mathrm{mcg}$ después de la ingestión del ABZ. Fue sugerido que el nivel sérico elevado de epinefrina y la reducción del flujo sanguíneo placentario pueden representar un riesgo potencial al feto. Estos efectos fueron confirmados para ratas por Kimmel et al. (1984). De la misma manera, Ross \& Persaud (1986) observaron reducción del flujo circulatorio, proliferación celular disminuida y grados variables de alteraciones degenerativas, que resultarían en alteración funcional, en las placentas de ratas tratadas con ABZ. Fujii \& Nichimura (1972) demostraron congestión marcada de los capilares de la placenta materna en ratas tratadas con ABZ.

Placentas menores con flujo sanguíneo disminuido, causan hipoxia fetal significativa, que provoca retardo de crecimiento intrauterino (Emmanouilides et al., 1972; Longo et al., 1978).

Es necesario recordar que los benzimidazoles causan reducción del consumo de alimentos en ratas grávidas (Ogata et al., 1984), y que las ratas desnutridas poseen placentas menores, con disminución significativa de los fosfolípidos y ácidos grasos libres, causando reducción de las funciones esenciales (Araya et al., 1986). Placentas menores fueron observadas en ratas que recibieron, durante la gravidez, mebendazol (Bombonato et al.) o SOABZ (Teruel et al.).

La longitud del cordón umbilical es un índice confiable del movimiento fetal, ya que es influenciado por dos factores: la incidencia de movimientos fetales y el espacio intrauterino disponible (Miller et al., 1981; Moessinger, 1983). Si ocurre disminución del espacio intrauterino o si el movimiento fetal se encuentra bloqueado, ocurre disminución del estiramiento del cordón umbilical, con reducción de su longitud.

Cordones más cortos fueron observados en ratas que, durante la gestación, fueron sometidas a restricción proteica, hipertermia, expuestas a la acción de alcohol, ciclofosfamida o metales pesados, o que recibieron dosis altas de vitamina A (Barron et al., 1986, Lopes et al., 1991/1992, Cunha et al., 1992, Oliveira et al., 1994, Liedtke et al., 1995). Nues- tros resultados revelaron cordones umbilicales más cortos en los animales tratados, indicando limitación del movimiento fetal.

A pesar de ser desconocido el volumen de líquido amniótico necesario para un crecimiento normal del feto, cualquier reducción de volumen (oligohidramnios) puede provocar anormalidades en ratas, como paladar hendido y deformaciones de los miembros (Symchych \& Winchester, 1978). La reducción del volumen de líquido amniótico, observada en este trabajo, podría indicar alteraciones en el crecimiento fetal y la aparición de malformaciones macroscópicas.

En el presente trabajo fue observada disminución del peso uterino en los animales tratados con ABZ. Reducción del peso uterino provocado por otros benzimidazoles en ratas fue también descrito por Cummings et al. (1990) y por Spencer et al. (1996). Estos hallazgos pueden ser explicados por el efecto de los benzimidazoles sobre el crecimiento tisular uterino y sobre la concentración proteica. El efecto directo sobre el útero, aparentemente no involucra mecanismos esteroides o de receptores (Spencer et al.), pero si ejerce sobre los canales de calcio (Yildirim et al., 2001).

En 1997, Piscopo et al., observaron que el SOABZ estaba presente en el líquido uterino de bovinos, lo que explicaría la causa de la susceptibilidad del embrión a los efectos letales de este metabolito durante el período de pre-implantación.

Los benzimidazoles y sus derivados, incluyendo el ABZ, son teratogénicos para ratas, ratones, conejos, porcinos, vacunos y ovinos, ocasionando malformaciones esqueléticas, como ectrodactilia, exencefalia, hidrocefalia, encefalocele, meningocele, onfalocele, malformaciones de las extremidades, anoftalmía, microftalmía, pie torcido (campto), atresia anal, ausencia de pálpebras, rabo corto, retorcido o ausente, labio leporino, paladar hendido, hidronefrosis y espina bífida (Lapras et al.; Szabo et al., 1974; Delatour et al., 1976; Wendler et al.; 1976, Khera et al.; Ogata et al., 1984; Yoshimura, 1987a; Baskerville et al.).

En este trabajo, administrando $5 \mathrm{mg} / \mathrm{kg}$ de ABZ, los días 9,10 y 11 de la preñez de rata, fueron observadas algunas reabsorciones, fetos menores, además de ausencia de rabo (40\%) y fetos hidrópicos (10\%). Con dosis de $10 \mathrm{mg} /$ $\mathrm{kg}$ se verificó el $100 \%$ de reabsorciones. Delatour et al. (1981) demostraron la presencia de malformaciones administrando 8,83 mg/kg de ABZ. Otros trabajos relatan ausencia de malformaciones con benomyl y triclabendazol (Yoshimura, 1987b), pero con retardo del crecimiento fetal en ratas, usando benomyl (Kavlock et al.), tiabendazol 
(Lankas \& Wise) o mebendazol (Bombonato et al.). El vehículo utilizado para disolver la droga desempeña papel importante en su acción. Así, el tiabendazol se mostró más teratogénico en suspensión de aceite de oliva (Ogata et al., 1984), que en solución acuosa a 0,5\% de goma arábiga (Ogata et al., 1987). De la misma manera, la captación de $14 \mathrm{C}$-tiabendazol por el feto, placenta y plasma, fue mayor cuando la droga estaba disuelta en aceite de oliva (Yoneyama et al., 1985).

En este trabajo fue observado que el hígado de los fetos tratados con ABZ estaba desorganizado, con hepatocitos menores y más abundantes, megacariocitos de volumen mayor y sinusoides dilatados en algunos fetos.

Los hepatocitos mostraron degeneración hidrópica y citoplasma granuloso. Algunas células estaban necrosadas y los núcleos en cariolisis. Los resultados morfométricos confirmaron esas observaciones. Los hepatocitos y sus núcleos son menores, justificando el mayor número de células por milímetro cúbico. Este cuadro indicaría que los hepatocitos no están diferenciándose de la misma manera en el feto tratado que en el feto control, resultando una hipotrofia. El menor volumen relativo de citoplasma y mayor para el núcleo resulta en una relación N/C aumentada en el feto tratado. El núcleo de los hepatocitos no mostró alteración de forma entre los animales tratados y controles.

Los megacariocitos hepáticos eran más numerosos en los fetos tratados que en los controles, traducido por el aumento de su volumen relativo. Además, eran mayores, como quedó demostrado por los resultados morfométricos. También el núcleo mostró valores mayores para perímetro, área y volumen, y su forma también estaba alterada.

A pesar que en algunos fetos los sinusoides se mostraron más dilatados, no hubo diferencia significativa del volumen relativo entre los dos grupos.

La concentración de linfocitos era semejante en los dos grupos de animales.

Los benzimidazoles presentan una tendencia a provocar rompimiento en las divisiones mitóticas en helmintos y hongos (Theodorides et al., 1976; Hammerschlag \& Sisler, 1977; Davidse \& Flach, 1978). Es necesario recordar que esa acción antimitótica y la inhibición de los complejos de la tubulina ocurren también en células de mamíferos (De Brabander et al., 1976; Friedman \& Platzer, 1978; Shandhu \& Waters, 1980). Se sabe también que la polimerización de la tubulina para formar microtúbulos es una función esencial en las células eucarióticas en los fe- nómenos estructurales, mitóticos, secretores y de transporte (Dustin, 1978; Roberts \& Hyams, 1979; Ireland et al., 1979). Esos efectos pueden, en parte, explicar la aparición de las malformaciones.

Los benzimidazoles pueden inhibir la síntesis de ATP en las mitocondrias (Allen \& Gottlieb, 1970; Tsuchiya \& Tanaka, 1985). Fue demostrado que el tiabendazol inhibe el transporte de electrones en las mitocondrias, impidiendo la liberación de energía y su almacenamiento en forma de ATP, dificultando la duplicación del DNA y la síntesis proteica (Allen \& Gottlieb). Estos hechos permiten explicar el cuadro de hipotrofia observado debido a la menor liberación de energía.

Probablemente, las malformaciones son causadas por la ligación de la droga a las macromoléculas en los tejidos (Yoneyama et al.). El inicio de la malformación puede involucrar un grado excesivo de mortalidad celular en los tejidos embrionarios, tornándolos, futuramente, malformados (Scott et al., 1971). Una dosis de tiabendazol puede matar pocas células al ligarse a macromoléculas fetales, mientras que altas dosis de droga causan la muerte de muchas células, resultando una disminución de la población celular bajo el punto crítico, provocando la malformación (Yoneyama et al.). En nuestro material fue posible verificar un gran número de células necrosadas (hepatocitos degenerados y con núcleos en cariolisis).

Tratando bovinos con SOABZ, Scarpelli et al. (1999) no demostraron reacción adversa después del tratamiento, ya que las funciones hepática y renal, además del hemograma, eran semejantes en los grupos de animales estudiados. Por otro lado, Locatelli et al. (2004) observaron condición de estrés oxidativo en el hepatocito de rata tratada con ABZ y mebendazol.

Los resultados obtenidos en este trabajo sugieren que la exposición prenatal al ABZ en dosis de $5 \mathrm{mg} / \mathrm{kg}$, actúa directamente en la organogénesis, causando malformaciones externas y modificaciones en el desarrollo, caracterizadas por reducción del peso corporal, peso de la placenta y longitud del cordón umbilical, así como por la desorganización generalizada del tejido hepático. El feto tratado, aún cuando no muestre malformaciones, presenta aspectos que sugieren inmadurez al ser comparado con el feto control.

AGRADECIMIENTOS: Los autores agradecen el apoyo financiero de la Universidad de Franca y del CNPq (Conselho Nacional de Desenvolvimento Científico e Tecnológico, Brasil). 
MORETI, D. L. C.; LOPES, R. A.; VINHA, D.; SALA, M. A.; SEMPRINI, M. \& FRIEDRICHI, C. Effects of albendazole on rat fetuses liver. Morphologic and morphometric studies. Int. J. Morphol., 23(2):111-120, 2005.

SUMMARY: The objective of the present work was to characterize morphological, morphometrical and stereologically the hepatic alterations in rat fetuses, caused by the administration of albendazole during pregnancy. They were obtained fetuses of Wistar rats, which received in days 9, 10 and 11 of pregnancy a daily dose of $5 \mathrm{mg} / \mathrm{Kg}$ body weight of albendazole. The control rats received injections of saline in the same days. At the 20th day, the rats were sacrificed by anesthetic ether inhalation, the fetuses were removed and immediately fixed in alfac for $24 \mathrm{~h}$. All the fetuses, uteri and placentas, after fixed, was dried in filter paper and weighed in a precision scale, whereas the umbilical cords were measured with a vernier. The bodies were longitudinally cut and embedded in paraffin. Six mm-thick serial sections were stained with hematoxylin and eosin. In addition to the histopatologic examination, histometric technics were used. The obtained results, in the used experimental conditions, suggest that prenatal exposition to albendazole directly acts on the embryogenesis, causing malformations, like agenesia of the tail and hydropic fetuses. In those fetuses without malformations, the drug acts on the fetal growth, causing modifications characterized by reduced body weight, placental weight and size of the umbilical cord, as well as structural alterations of the hepatic tissue, translated by smaller hepatocytes with smaller nuclei, and scanty cytoplasm but increased number density. The megacariocytes were more volumous, with greater lobulated nuclei, occupying a higher relative volume. The sinusoids occupied a similar relative volume in the fetuses of both studied groups. The treated fetuses, in addition to the malformations appeared to be immature, when compared to the controls.

KEY WORDS: Liver; Fetus; Rat; Albendazole; Morphometry.

\section{REFERENCIAS}

Allen, P. \& Gottlieb, D. Mechanism of action of the fungicide thiabendazole, 2-(4-thiazolyl) benzimidazole. App. Microbiol., 20(6):919-28, 1970.

Araya, J.; Aguilera, A. M.; Soto, C. \& Masón, L. Composición lipídica de la placenta de ratas con restricción de proteinas y deficiencia de ácidos grasos esenciales. Arch. Latinoam. Nutr., 36(2):237-337, 1986.

Barron, S.; Riley, E. P. E. \& Ptherman, W. P. The effect of prenatal alcohol exposure on umbilical cord length in fetal rats. Clin. Exp. Res., 10(5):493-5, 1986.

Baskerville, M.; Wood, M. \& Newton, C. M. Mebendazole for worming mice: Effectiveness and side effects. Lab. Anim., 22(2):263-8, 1988.

Bombonato, K. F.; Prata, G.V.; Brentegani, L. G.; Sala, M. A.; Vale, S. A. L. \& Lopes, R. A. Estudo morfológico de fetos de ratas injetadas com mebendazol. Rev. Reg. Ciênc., 3(1):19-24, 1994.

Bradley, M. \& Horton, J. Assessing the risk of benzimidazole therapy during pregnancy. Trans. Roy. Soc. Trop. Med. Hyg., 95(1):72-3, 2001.

Chalkley, H. W. Method for the quantitative morphologic analysis of tissues. J. Natl. Cancer Inst., 4(1):47-53, 1943.
Cristófol, C.; Navarro, M.; Franquelo, C.; Valladares, J.E.: Carretero, A.; Ruperto, J. \& Arboix, M. Disposition of netobimin, albendazole, and its metabolites in the pregnant rat developmental toxicity. Toxicol. Appl. Pharmacol., 144(1):56-61, 1997.

Cummings, A. M.; Harris, S. T. \& Rehnbeerg, G. L. Effects of methyl benzimidazole carbamate during early pregnancy in the rat. Fundam. Appl. Toxicol., 15(3):52835, 1990.

Cunha, S. A.; Contrera, M. G. D.; Lopes, R. A.; Azoubel, R. \& Contrera, J. D. Experimental hypervitaminosis A in the rat. XXV. The effect of a high single dosis of vitamin A on umbilical cord length. Anais $44^{a}$ Reunião SBPC, 1(1):712, 1992.

Davidse, L.C. \& Flach, W. Interaction of thiabendazole with fungal tubulin. Biochim. Biophys. Acta, 543(1):82-90, 1978.

De Brabander, M. J.; Van de Veire, R. M. L.; Aerts, F. E. M.; Borgers, M. \& Janssen, P. A. J. The effects of R 17934 (NSC 238159), a new synthetic antitumoral drug interfering with microtubules, on mammalian cells cultured in vitro. Cancer Res., 6(3):905-16, 1976.

Delatour, P.; Daudon, M.; Garnier, F. \& Benoit, E. Relationship of metabolism and embryotoxicity of 
febantel in the rat and sheep. Ann. Rech. Vet., 13(2):16370, 1982.

Delatour, P.; Garnier, F.; Benoit, E. \& Longin, C. H. A correlation of toxicity of albendazole and oxfendazole with their free metabolites and bound residues. J. Vet. Pharmacol. Ther., 7(1):139-45, 1984.

Delatour, P.; Lorgue, G.; Lapras, M. \& Richard, Y. Propiétés embryotoxiques du parbendazole, du mébendazole et du cambendazole. C. R. Acad. Sci., Paris, 282 (5):5178, 1976.

Delatour, P.; Parish, R. C. \& Gyurik, R.J. Albendazole: a comparison of relay embryotoxicity of individual metabolites. Ann. Res. Vét., 12(2):159-67, 1981.

Duncan, W. A .M. \& Lemon, P. G. The effects of methyl5(6)-butyl-20benzimidazole carbamate (parbendazole) on reproduction in sheep an other animals. Part VIII. Cornell Vet., 64(Suppl. 4):97-103, 1974.

Dustin, P. Microtubules. Berlin, Springer, 1978.

Emmanouilides, G. C.; Hobel, C. J.; Vashiro, K. \& Klyman, $\mathrm{G}$. Fetal responses to maternal exercise in the sheep. Am. J. Obstet. Gynecol., 112(1):130-7, 1972.

Fabre, J. M.; Berthelot, X. \& Ferney, J. Embryotoxicité des antiparasitaires chez les ovins. Rev. Méd. Vét., 140(12): 1098-95, 1989.

Friedman, P. A. \& Platzer, E. G. Interaction of anthelmintic benzimidazole derivatives with bovine brain tubulin. Biochem. Biophys Acta, 544(3):605-14, 1978.

Fujii, T. \& Nichimura, A. Adverse effects of prolonged administration of caffeine on rat fetus. Toxicol. Appl. Pharmacol., 22(2): 449-57, 1972.

Goldstein, A.; Aronow, L. \& Kalman, M. S. M. Principles of drug action. New York, Harper and Row, 1968.

Hammerschlag, R. S. \& Sisler, H. D. Benomyl and methyl2-benzimidazolecarbamate (MBC): biochemical, cytological and chemical aspects of toxicity to Ustilago maydis and Saccharomyces cerevisiae. Pestic. Biochem. Physiol., 3(1):42-54, 1977.

Huggett, A. S. G. \& Hammond, J. Physiology of the placenta. In: Parkes, A.S. (ed.) Marshall's Physiology of Reproduction. 3. ed. London, Longmans, Green \& Co., 1952.
Ireland, C. M.; Gull, K.; Gutteridge, W. E. \& Podson, C. I. The interaction of benzimidazole carbamates with mammalian microtubule protein. Biochem. Pharmacol., 28(10):2680-2, 1979.

Kavlock, R. J.; Chernoff, N.; Gray Jr, L. E.; Gray, J. A. \& Whitehouse, D. Teratogenic effects of benomyl in the Wistar rat and CD-1 mouse, with emphasis on the route of administration. Toxicol. Appl. Pharmacol., 62(1):4454, 1982.

Khera, K. S.; Whalen, C.; Trivett, G. \& Angers, G. Teratologic assessment of maleic hydrazide and daminozide and formulations of ethoxyquin, thiabendazole and naled in rats. J. Environ. Sci. Health, 14 (6):563-77, 1979.

Kimmel, C. A.; Kimmel, C. L.; White, C. G.; Grafton, T. F.; Yong, J. F. \& Nelxom, C. J. Blood flow changes and conceptal development in pregnant rats in response to caffeine. Fund. App. Toxicol., 4(2):240-7, 1984.

Kirkinem, P.: Jouppila, P.; Koivula, A.; Vuori, J. \& Puukka, M. The effect of caffeine on placental and fetal blood flow in human pregnancy. Am. J. Obstet. Gynecol., 147(8):939-42, 1983.

Lankas, G. R. \& Wise, D. L. Developmental toxicity of orally administered thiabendazole in Sprague-Dawley rats and New Zealand white rabbits. Fed. Chem. Toxicol., 31(3): 199-207, 1993.

Lanusse, C. E. \& Pritchard, R.K. Relationship between pharmacological properties and clinical efficacy of ruminant anthelmintics. Vet. Parasitol., 49(2-4):12358, 1993.

Lapras, M.; Delatour, P.; Deschanel, J.; Lourge, G.; Camps, D. \& Regnier, B. Étude expérimentale de l'activité tératogéne du parbendazole chez le rat. Bull. Soc. Sci. Vét. Méd. Comp., 75(1):117-30, 1973.

Liedtke Jr., H.; Azoubel, R.; Tubino, P. J. G. \& Lopes, R. A. Estudo morfológico de fetos de ratas tratadas com ciclofosfamida durante a gestação. Rev. Reg. Ciênc., 4(1):95-104, 1995.

Locatelli, C.; Pedrosa, R.C.: De Bem, A.F.; CreczunskiPasa, T. B.; Cordova, C. A. \& Wilhelm-Filho, D. A comparative study of albendazole and mebendazoleinduced, time-dependent oxidative stress. Redox Rep., 9(2):89-95, 2004. 
Longo, L.; Hewitt, C. W.; Lorijn, R. H. \& Gilbert, R .D. To what extent does maternal exercise affect fetal oxygenation and uterine blood flow? Fed. Proc., 37(3):905, 1978.

Lopes, R. A.; Sala, M. A.; Maia Campos, G.; Petenusci, S. O. \& Komesu, M. C. The effect of methylmercury on umbilical cord length. An. Farm.Quim., 31(Supl.):66, 1991/1992.

Makita, T.; Hashito, Y. \& Nouguchi, T. Mutagenic, cytogenic and teratogenic studies on thiophanate-methyl. Toxicol. Appl. Pharmacol., 24(1):206-15, 1973.

Mantovani, A.; Ricciardi, C.; Stazi, A.V. \& Macri. C. Effects observed on gestational day 13 in rat embryos exposed to albendazole. Reprod. Toxicol., 9(3):265-73, 1995.

Marriner, S. Anthelmintic drugs. Vet. Rec., 118(1):181-4, 1986.

McKellar, G. \& Scott, E. The benzimidazole anthelmintic agents - a review. J. Vet. Pharmacol. Ther., 13(1):22347, 1990 .

Merz, W. A. Streckenmessung an gerichteten Strukturen im Mikroskop und anwendung zur Bestimmung von Oberflachen Volumen relationen im Knochengewebe. Mikroskopie, 22(5-6):132-42, 1968.

Miller, M. E.; Higginbottom, M. \& Smith, D.W. Short umbilical cord, its origin and relevance. Pediatrics, 67(5):618-21, 1981.

Moessinger, A. C. Fetal akynesia deformation sequence: an animal model. Pediatrics, 72(6):857-63, 1983.

Navarro, M.; Canut, L.; Carretero, M.; Arboix, M. \& Buberje, J. Developmental toxicity in rat fetuses exposed to the benzimidazole netobimin. Reprod. Toxicol., 13(2):295302, 1999.

Ogata, A.; Yoneyama, M.; Sasaki, M.; Susuki, K. \& Imamichi, T. Effects of pretreatment with SKF-525 A or sodium phenobarbital on thiabendazole-induced teratogenicity in ICR mice. Fed. Chem. Toxicol., 25(2):119-24, 1987.

Ogata, A.; Ando, H.; Kubo, Y. \& Hiraga, K. Teratogenicity of thiabendazole in ICR mice. Fed. Chem. Toxicol., 22(7):509-20, 1984.

Oliveira, P. T.; Lopes, R. A.; Toneto, A. D. R.; Sala, M. A.; Maia Campos, G. \& Watanabe, I. The effect of hyperthermia on umbilical cord and placenta. An. Farm. Quim., 33 (1):33-4, 1994.

Piscopo, S. E. \& Smoak, I.W. Comparison of effects of albendazole sulfoxide on in vitro produced bovine embryos and rat embryos. Am. J. Vet. Res., 58 (9):103842, 1997.

Roberts, K. \& Hyams, J.S. Microtubuli. New York, Academic Press, 1979.

Ross, C. P. \& Persaud, T. V. N. Early embryonic development in the rat following in utero exposure to alcohol and caffeine. Histol. Histopathol., 1(1):13-7, 1986.

Sala, M. A.; Komesu, M. C.; Lopes, R. A. \& Maia Campos, G. Karyometric study of basal cell carcinoma. Braz. Dental J., 5(1):11-4, 1994.

Sala, M. A.; Lopes, R. A. \& Matheus, M. Método morfológico para análisis cuantitativo de los tejidos. Determinación de los parámetros normales para el hepatocito de rata. Arch. Fac. Med. Zaragoza, 32(1):29-31, 1992.

Scarpelli, L. C.; Belo, M. A. A.; Souza, L. M.; Sabatini, G. A.; Costa, A. J. \& Moraes, F.R. Parâmetros hematológicos e bioquímicos de bovinos submetidos a diferentes doses de sulfóxido de Albendazole, Ivermectin e Abamectin. Ars Veter. (Supl.):57-62, 1999.

Scott, J.W.; Ritter, J.E. \& Wilson, J.G. DNA synthesis inhibition and cell death associated with hydroxyurea teratogenesis in rat embryos. Dev. Biol., 26(2):306-15, 1971.

Shandhu, S.S. \& Waters, M.D. Mutagenicity evaluation of chemical pesticides. J. Environm. Sci. Health B., 15(12): 929-48, 1980.

Spencer, F.; Chi, L. \& Zhu, M.-X. Effect of benomyl and carbendazin on steroid and molecular mechanisms in uterine decidual growth in rats. J. Appl. Toxicol., 16(3): 211-4, 1996.

Symchych, P. S. \& Winchester, P. Potter's syndrome. Animal model: amniotic fluid defficiency and lung growth in the rat. Am. J. Pathol., 90(3):779-82, 1978.

Szabo, K. T.; Miller, C. R. \& Scott, G. C. The effect of methyl 5(6)-butyl-2-benzimidazole carbamate (parbendazole) on reproduction in sheep and other animals. II. Teratological study in reproduction in ewes in the United States. Cornell Vet., 64(4):41-55, 1974. 
Teruel, M. T.; Felipe, A. E.; Solana, H. D.; Sallovitz, J. M. \& Lanusse, C. E. Placental and fetal toxicity of albendazole sulphoxide in Wistar rats. Vet. Human Toxicol., 45(3):1316, 2003.

Theodorides, V. J.; Gyurik, R.; Kingsbury, W.D. \& Parish, R. C. Anthelmintic activity of albendazole against liver flukes, tapeworms, lung and gastrointestinal roundworms. Experientia, 32(6):702-3, 1976.

Tsuchiya, T. \& Tanaka, A. In vivo inhibition of adenosine triphosphate (ATP) synthesis associate with thiabendazoleinduced teratogenesis in mice and rats. Arch. Toxicol., 57(4):243-5, 1985.

Wendler, V. D.; Pabst, R. \& Bertolini, R. Über die Beeinflussung von Schwangerschaft und Keimlingsentwicklung der Ratte durch das N-lost-derivat "Cytostasan". Anat. Anz., 139(2):110-4, 1976.

Yildirim, K.; Sarioglu, Y.; Kaya, T.; Cetil, A. \& Yildirim, S. Inhibitor effect of omeprazole in isolated human myometrial smooth muscle. Life Sci., 69(4):435-44, 2001.

Yoneyama, M.; Ogata, A. \& Hiraga, K. Irreversible in vivo binding of thiabendazole to macromolecules in pregnant mice and its relation to teratogenicity. Food Chem. Toxicol, 23(8):733-6, 1985.

Yoshimura, $\mathrm{H}$. Teratogenic evaluation of triclabendazole in rats. Toxicology, 43 (3):283-7, 1987b.

Yoshimura, H. Teratogenicity of flubendazole in rats. Toxicology, 44(2):133-6, 1987a.

Zhangliu, C.; Zhenling, Z.; Zhonghul, Y. \& Rifai, F. Pharmacokinetics of albendazole in pigs. J. South China Agric. Univ., 11(3):51-6, 1992.
Dirección para correspondencia:

Prof. Dr. Ruberval A. Lopes

Faculdade de Odontologia de Ribeirão Preto - USP

Universidade de Såo Paulo

Av. do Cafe, s/n

CEP 14040-904

Ribeirão Preto-SP

BRASIL

e-mail: ralopes@forp.usp.6r

Recibido : 22-02-2005

Aceptado: 21-04-2005 